\title{
Transcatheter mitral valve repair using the MitraClip: which patients benefit most?
}

\author{
Julia Mascherbauer
}

(C) Springer-Verlag GmbH Austria, part of Springer Nature 2018

Surgical treatment of primary mitral regurgitation (MR) is well-established [1] and controversies only remain with respect to the timing of surgery (early invasive versus watchful waiting). Such considerations, however, only apply to the operable individual with primary MR. As the life expectancy of the general population has significantly increased in recent years, more and more 80-90-year-old patients are diagnosed with primary MR. In addition, a considerable proportion of patients with primary MR are deemed inappropriate candidates for surgery due to frailty, previous cardiac surgery, porcelain aorta or other factors. Although systematic data with respect to the use of transcatheter mitral valve repair with the MitraClip device in patients with primary MR are currently missing it seems very likely that this population is excellently suited to undergo such a minimally invasive procedure and that these patients will significantly benefit.

With respect to functional MR, much more controversy currently exists as to whether these patients should be treated at all and at what time point in the disease history. The presentation of the results of the COAPT [2] and the Mitra-FR [3] trials has not resolved but aggravated these controversies. Treatment of functional MR has remained controversial despite many years of surgical, medical and more recently, catheter-based options. Surgical annuloplasty of functional MR at the time of coronary bypass surgery is well-established and has received a class IC recommendation in the current guidelines [1]. Isolated surgical annuloplasty for functional MR in heart failure patients may be considered (class IIbC indica-

Univ.-Prof. Dr. J. Mascherbauer ( $₫)$

Department of Cardiology, Medical University of Vienna, Waehringer Guertel 18-20, 1090 Vienna, Austria

julia.mascherbauer@meduniwien.ac.at tion) [1] but has not yet proven superior compared with conservative treatment in randomized controlled trials.

The MitraClip device may be a game-changer in this specific patient population. After approximately 10 years of use and more than 60,000 implantations worldwide, the COAPT trial for the first time showed a huge survival benefit after 2 years in heart failure patients with significant functional MR who were treated with the MitraClip [2]. In the COAPT trial 614 heart failure patients with significant MR from 78 American and Canadian centers were randomly assigned to MitraClip in addition to optimal medical treatment (device group) or optimal medical treatment alone (control group). An independent echocardiographic core laboratory had to confirm MR severity, left ventricular systolic function (required to range from $20 \%$ to $50 \%$ ejection fraction), left ventricular dimensions, and a high probability that the mitral valve could be repaired with the MitraClip system. Patients were only eligible if they were symptomatic despite optimal medical treatment with a New York Health Association (NYHA) score $\geq 2$. The local heart team had to deem surgery inappropriate. The primary end-point in terms of effectiveness was all-cause hospitalization for heart failure at 2 years.

302 patients were assigned to the device group, and 312 to the control group. At 2 years, the primary endpoint was significantly more often met in the control group (hazard ratio, 0.53 ; 95\% confidence interval, $0.4-0.7 ; p<0.0001)$. Furthermore, all-cause mortality was reduced by $17 \%$ in the device-group (hazard ratio 0.62, number needed to treat at 24 months 5.9). These results indicate a major advancement in the treatment of heart failure. A recent meta-analysis including 2121 heart failure patients confirmed the findings of COAPT by demonstrating a significant survival benefit in those treated with the MitraClip device [4]. 
Apart from the benefits with respect to heart failure hospitalization and death, small series have shown that a decrease of pulmonary pressures after reducing MR severity with the MitraClip device rendered endstage heart failure patients eligible for heart transplantation who previously had been rejected due to inappropriate hemodynamics [5].

Why, however, were the fabulous results of COAPT not seen at all in the Mitra-FR trial? This is one of the main controversies among heart failure specialists right now. The Mitra-FR trial randomized 304 heart failure patients with significant MR at 37 centers in France to either MitraClip implantation in addition to optimal medical therapy (device group) or optimal medical treatment alone (control group). All echocardiograms were evaluated in a core laboratory that had to confirm MR severity and that left ventricular ejection fractions ranged from $15 \%$ to $40 \%$. Similarly to COAPT, surgery had to be deemed inappropriate by the local heart team. Follow-up was shorter than in COAPT-12 months in comparison to 24 months - and the primary endpoint was a composite of unplanned hospitalization for heart failure and all-cause death. In the study 152 patients were randomly assigned to each group. After 1 year, no difference in the primary endpoint was observed between the device and control groups $(p=0.53)$.

Since presentation of both trials, numerous potential explanations for the differences in findings in COAPT and Mitra-FR have been put forward. Among them are considerations concerning inclusion criteria and background medical therapy. The COAPT trial enrolled patients between 2012 and 2017, Mitra-FR between 2013 and 2017 but only with half as many patients. Patients on medical treatment improved in Mitra-FR during the study period (patient-study benefit?) while they did not in COAPT.

Certainly, further (sub)analyses of both trials and future upcoming studies will shed more light on the most important remaining question: which are the patients who will benefit most from MitraClip implantation?-Those with primary MR deemed inoperable?-End-stage heart failure patients not eligible for heart transplantation due to high pulmonary pressures?-Among heart failure patients with secondary MR those who have a more advanced disease or a less advanced disease? Exciting times are ahead for the MitraClip community.

Conflict of interest J. Mascherbauer declares that she has no competing interests.

\section{References}

1. Baumgartner H, Falk V, Bax JJ, De Bonis M, Hamm C, Holm PJ, Iung B, Lancellotti P, Lansac E, Rodriguez Muñoz D, Rosenhek R, Sjögren J, Tornos Mas P, Vahanian A, Walther $\mathrm{T}$, Wendler O, Windecker S, Zamorano JL, ESC Scientific Document Group. 2017 ESC/EACTS Guidelines for the management of valvular heart disease. Eur Heart J. 2017;38(36):2739-91.

2. Stone GW, Lindenfeld J, AbrahamWT, Kar S, Lim DS, Mishell JM, Whisenant B, Grayburn PA, Rinaldi M, Kapadia SR, RajagopalV, SarembockIJ, BriekeA, MarxSO, Cohen DJ, Weissman NJ, Mack MJ, COAPT Investigators. Transcatheter mitral-valve repair in patients with heart failure. $\mathrm{N}$ Engl J Med. 2018; https://doi.org/10.1056/NEJMoa1806640.

3. Obadia JF, Messika-Zeitoun D, Leurent G, Iung B, Bonnet G, Piriou N, Lefèvre T, Piot C, Rouleau F, Carrié D, Nejjari M, Ohlmann P, Leclercq F, Saint Etienne C, Teiger E, Leroux L, Karam N, Michel N, Gilard M, Donal E, Trochu JN, Cormier B, Armoiry X, Boutitie F, Maucort-Boulch D, Barnel C, Samson G, Guerin P, Vahanian A, Mewton N, MITRA-FR Investigators. Percutaneous repair or medical treatment for secondary mitral regurgitation. NEngl J Med. 2018; https:/ / doi.org/10.1056/NEJMoa1805374.

4. Giannini C, D'ascenzo F, Fiorelli F, Spontoni P, Swaans MJ, Velazquez EJ, Armeni P, Adamo M, De Carlo M, Petronio AS. A meta-analysis of MitraClip combined with medical therapy vs. medical therapy alone for treatment of mitral regurgitation in heart failure patients. ESC Heart Fail. 2018; https://doi.org/10.1002/ehf2.12339.

5. Crimi G, Gritti V, Ghio S, Crescio V, Magrini G, Scelsi L, Turco A, Gazzoli F, Ferrario M, Raineri C, Oltrona Visconti L. MitraClip procedure as 'bridge to list', the ultimate therapeutic option for end-stage heart failure patients not eligible for heart transplantation due to severe pulmonary hypertension. Pulm Circ. 2018; https://doi.org/10.1177/ 2045894018791871. 